\title{
EL SECTOR PRIVADO DE LA ECONOMIA SOVIETICA EN LOS AÑOS VEINTE
}

\author{
LEONID BORODKIN Y MIJAIL SVISCHOV \\ Universidad de Moscú
}

\section{RESUMEN}

El artículo estudia la movilidad social de los propietarios privados bajo la NEP (Nueva Política Económica) en la Unión Soviética durante los años veinte. La cuestión planteada es si era posible un rápido crecimiento de los sectores capitalistas bajo las condiciones sociales y económicas existentes. Más precisamente, se propone contrastar la idea de si la economía de mercado conlleva la diferenciación entre 108 propietarios privados y la formación de grupos sociales antagónicos a partir de una masa homogénea de pequeños productores de mercancias. Para ello se emplea un modelo de simulación basado en las cadenas de Markov y los resultados muestran la dirección y entidad de la diferenciación social y sugieren que, de haber prevalecido la NEP, los propietarios privados no se habrian desintegrado en grupos opuestos.

\section{ABSTRACT}

This article deals with the social mobility of private owners under the $\mathrm{N}(\mathrm{ew}) \mathrm{E}$ (conomic) $\mathrm{P}(\mathrm{olicy})$ in the Soviet Union during the 1920's. The question addressed is: Was it possible a rapid growth of capitalist stratra under the prevalent social and economic conditions? More specifically, the paper puts to the test the idea that market economy inevitably entails private owners differentiation and the formation of antagonistic social groups from the formerly homogeneous mass of petty commodity producers. A simulation model based on Markov chains was applied and its results show the directions and extent of social differentiation suggesting that had the NEP survive, private owners would not have disintegrated into opposed groups.

La revolución de octubre de 1917 en Rusia se llevó a efecto bajo las consignas de igualdad social y no explotación del hombre por el hombre. Los bolcheviques que arribaron al poder a resultas de la sublevación popular, vieron la vía para alcanzar estos objetivos, democráticos por su carácter, en la socialización de los medios de producción. Seguian la teoría según la que la acción de las leyes de mercado lleva indefectiblemente a una estratificación de 
la masa, otrora homogénea, de los pequeños productores. Su resultado es la concentración en los polos opuestos de los propietarios de los medios de producción y de los propietarios de la única mercancía, fuerza de trabajo. Así surge la desigualdad social y la explotación como consecuencia del trabajo asalariado. De estas posturas se deduce la urgencia de acabar con la propiedad privada de los medios de producción y con las relaciones mercantiles monetarias.

El partido bolchevique, al convertirse en el partido gobernante, no escatimó esfuerzos para hacer realidad sus ideas. La política de ucomunismo de guerra» que se estaba configurando durante la guerra civil apuntaba, al fin y al cabo, a crear una economía donde las relaciones mercantiles monetarias serían reemplazadas por un intercambio directo de productos y una distribución administrativa. La destrucción de los lazos económicos tradicionales acompañada por las desgracias engendradas por las guerras, que duraron siete largos años, llevaron al pais al borde de una catástrofe estrechando al máximo la base social del poder bolchevique. Los campesinos, privados de la independencia, perdieron el estímulo a la ampliación y el mejoramiento de la hacienda. Se sembraba el mínimo para asegurar la subsistencia. En su totalidad la experiencia del "comunismo de guerra" demostró que la pequeña producción, siendo entonces la base de la economía nacional, podia funcionar tan sólo en las condiciones de mercado. A instancias de Lenin, la política económica experimentó un cambio cardinal: los campesinos obtuvieron el derecho de poseer lo producido, se admitió la pequeña empresa en la industria y el comercio privado que antes existía clandestinamente. Desde el punto de vista del objetivo estratégico - crear una sociedad sin la propiedad privada - la nueva política económica (NEP - de las siglas rusas) fue un paso hacia atrás respecto del "comunismo de guerra". Fue vista por los bolcheviques como una retirada, una maniobra politica. Se discutia tan sólo la cuestión de cuánto tiempo duraría esta retirada.

Puesto que se legalizaron las relaciones mercantiles monetarias, habria de comenzar, de acuerdo con la teoría, la división de los pequeños productores, es decir, de la mayoría de la población (campesinos, artesanos, pequeños productores). Conforme a esto se reforzarían las posiciones sociales y económicas de los grupos posesores llevando a que en cierta etapa el balance de fuerzas politicas se inclinaría a su favor. Tras ello tendrían que pasar cambios en el régimen politico. Lenin hizo el balance de estos razonamientos con una sola frase: «La libertad de comercio significa la retirada hacia el capitalismo» ${ }^{1}$.

I V. I. Lenin (1975), p. 61. 
El temor ante una "burguesia nueva" que se estaba formando a resultas de la diferenciación entre los pequeños productores, ante el «capitalismo creciente», fue uno de los resortes importantes de la política económica de los años 20. En fin de cuentas, llevó a la colectivización forzada de la agricultura a comienzos de los años 30, lo que privó de la independencia económica a los campesinos, a la vez que se prohibió la empresa privada en la industria y el comercio.

La tesis de que la pequeña producción mercantil representaba una amenaza para el socialismo se hizo base teórica de toda la historiografía soviética dedicada al estudio de los años 20 y 30 . Al mismo tiempo, prácticamente no se estudió el plano histórico concreto de la estratificación del sector privado de la economia nacional. Primero, porque parecía que esa tesis no requiere argumentación complementaria por estar incluida orgánicamente en el sistema común de ideas que interpretaban el socialismo como un régimen con la economia no mercantil, con la denominación, juridicamente asegurada, de la propiedad estatal y el dictado desde el centro sobre el productor. Segundo, es mucho más complicado analizar la dinámica de los procesos sociales que la estructura social. Para ello los historiadores poseen menos cantidad de fuentes y son menos perfectos los métodos de su análisis. Estas causas llevaron a que los científicos centraron su atención en la estructura social de la sociedad soviética, interpretando la presencia de diferentes grupos económicos dentro de la clase de productores pequeños como un signo de la diferenciación. $O$ sea, el estudio real de la dinámica fue reemplazado por los razonamientos al respecto basados en el análisis del cuadro estadístico.

La práctica económica obligó a cambiar los puntos de vista que se establecieron en los estudios soviéticos sobre las perspectivas del desarrollo de diferentes formas de organización de la producción. La pequeña empresa en todo el mundo, lejos de desaparecer de acuerdo con la teoria, está demostrando una flexibilidad extraordinaria, una capacidad de adaptarse a la coyuntura, de utilizar los logros de la revolución científico-técnica. La necesidad de este elemento estructural para el funcionamiento normal de la economía es demostrada por el desequilibrio económico de los países donde es limitado artificialmente el desarrollo de la pequeña empresa. Ello obliga a pensar que la tesis sobre una estratificación inevitable de los pequeños productores, o sea, la tesis que servía de base para el pensamiento político y la práctica económica de los años 20 requiere una revisión. En sus marcos hace falta determinar la estructura social del sector privado de la economía nacional que podría configurarse si durante varios años más hubiera existido la pequeña producción en desarrollo, tal como existia durante la NEP. De 
este modo, la tarea se reduce a analizar una situación que sea contraria a la existente, a tratar de reproducir una variante de alternativa en el desarrollo de procesos sociales y económicos durante la década del 1930. El instrumento para resolver el problema será el modelado de imitación basado en la teoria de las cadenas de Markov.

\section{EL CARACTER DE LA DINAMICA SOCIAL EN EL COMERCIO PRIVADO A MEDIADOS DE LOS AÑOS 20}

Según opinan los sociólogos soviéticos, el proceso de estratificación de los pequeños productores pasa en su desarrollo por tres etapas. En la primera, en su seno surge una desigualdad económica y se separan unas capas sustancialmente diferentes desde el punto de vista económico productivo, aunque en lo social-económico los pequeños productores siguen siendo unidos. Son móviles los límites que separan las capas, de ahí que es grande la posibilidad de paso de una categoría a la otra. El carácter asi de estratificación es intrínseco al momento inicial de génesis del capitalismo. En la segunda fase surge una estratificación social, capitalista, se forman unos grupos estables, opuestos por su posición. Al mismo tiempo sigue existiendo y predominando numéricamente la capa media. Durante esta etapa son posibles, por lo general, los pasos de la capa media a la inferior y, en pequeña proporción, a la superior. Es muy baja la posibilidad de paso de la capa inferior a la superior y viceversa. En la tercera fase se desintegra la capa media, y la masa de pequeños productores, que no hace mucho fue socialmente homogénea, se divide en dos grupos opuestos presentando como resultado una división social propia de un determinado nivel de desarrollo del capitalismo. De ahí que sea posible estudiar el proceso de diferenciación basándose en los datos de desplazamientos sociales. Su carácter, dirección e intensidad permiten formarse una idea de la fase que alcanzó la estratificación de los pequeños productores.

El presente artículo está dedicado a la dinámica social de los comerciantes y los empresarios. El interés por este grupo es condicionado por la diferenciación más rápida que se produce en las esferas de intercambio y artesanía en comparación con la de agricultura. Por eso el pronóstico retrospectivo de la estructura social de comerciantes y empresarios, basado en el modelado matemático, demuestra el nivel de estratificación máximo para una situación dada. Representa una especie de limite potencial para el crecimiento capitalista a condición de conservarse el sistema económico de la NEP. 
La información acerca de los cambios operados en el status social y de propiedad de los dueños de empresas comerciales e industriales fue recogida durante una investigación del sistema de impuestos en Ucrania, realizada en 1926-272. Según la ley, los empresarios estaban obligados a adquirir anualmente permisos especiales (patentes) en los organismos financieros locales, lo que les daba el derecho de realizar la actividad comercial. Según el tamaño de las empresas y de la cantidad de sus trabajadores éstas se dividian en cinco categorías ${ }^{3}$. Durante la indagación los inspectores de hacienda fijaron el tipo de patente que poseían los empresarios el año anterior. En total pudieron recoger datos acerca del 78,9 por 100 de las empresas privadas en acción. Así que los datos de la investigación realizada por los organismos fiscales permiten ver los cambios en las categorias de patentes de 1925-26 a 1926-27. Dichos cambios pueden relacionarse con los desplazamientos de los propietarios de un grupo social a otro.

Las patentes de primera y segunda categorias las tenian que adquirir los mercaderes ambulantes, los propietarios de kioscos y tiendas en los mercados, los pequeños acaparadores de los productos agricolas. Entre ellos tan sólo el 0,3 por 100 utilizaban mano de obra asalariada, mientras que la parte de trabajo asalariado en la estructura de ocupación fue del 0,4 por 100 . Los propietarios de pequeños almacenes que realizaban el comercio al por mayor adquirían patentes de tercera categoria. La parte relativa del trabajo asalariado alli fue del 9,1 por 100 . Los propietarios de almacenes grandes y los mercaderes al por mayor tenían que elegir patentes de cuarta o quinta categoría. El 46,9 por 100 de dichas empresas utilizaban la mano de obra asalariada cuya parte relativa en la estructura de ocupación fue del 32,1 por 100 (véase tabla 1). De esta manera, el paso de un mercader de primera o segunda categoría a la cuarta o quinta significaba que se convertía en un empresario de tipo capitalista, mientras que la disminución de la parte de patentes de tercera categoría era indicio de erosión de la capa media, etc.

Hay que indicar que el concepto de «empresa de tipo capitalista" debe

2 En la URSS toda la planificación en los años 20 se realizaba sobre la base de los llamados xaños económicos» que comenzaban el 1 de octubre. Por ejemplo, el año económico 1926-27 duró desde el 1 de octubre de 1926 hasta el 31 de septiembre de 1927.

3 De acuerdo con la ley sobre la imposición empresarial (1923), la patente de primera categoría se adquiria para realizar el comercio en los mercados utilizando un cesto de mimbre por persona; la de la segunda, para el comercio con ayuda de un miembro de familia en kioscos, casillas, tiendas; la de la tercera, para el comercio minorista con participación de no más de cuatro personas; la de la cuarta, para el comercio minorista con participación de 5 a 10 personas, y para el comercio semimayorista con ayuda de no más de 7 personas; la de la quinta, para el comercio minorista con participación de más de 7 personas y para el comercio al por mayor. 
entenderse en el contexto del comercio privado de los años 20. Las empresas comerciales no eran grandes. Ni las empresas de quinta categoría tenían más de seis ocupados y más de tres asalariados. Nunca era menos del 60 por 100 la parte de trabajo propio de los propietarios, copropietarios y miembros de sus familias.

TABLA 1

El trabajo asalariado en el comercio privado*

\begin{tabular}{|c|c|c|c|c|c|c|}
\hline \multirow{2}{*}{$\begin{array}{c}\text { Categorias } \\
\text { de }\end{array}$} & \multicolumn{6}{|c|}{ Indicadores } \\
\hline & 1 & 2 & 3 & 4 & 5 & 6 \\
\hline$\ldots \ldots \ldots \ldots \ldots \ldots \ldots \ldots \ldots \ldots$ & 15.503 & 4 & 0,03 & 15.725 & 6 & 0,04 \\
\hline 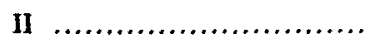 & 31.027 & 139 & 0,45 & 36.473 & 182 & 0,50 \\
\hline $\mathrm{I}+\mathrm{II} \ldots \ldots \ldots \ldots \ldots \ldots \ldots \ldots$ & 46.530 & 143 & 0,31 & 52.198 & 188 & 0,36 \\
\hline 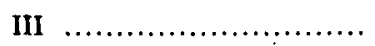 & 20.619 & 2.600 & 12,61 & 36.242 & 3.292 & 9,08 \\
\hline IV ... & 2.051 & 842 & 41,05 & 5.770 & 1.663 & 28,82 \\
\hline $\mathrm{V} \ldots \ldots$ & 398 & 307 & 77,14 & 2.472 & 987 & 39,93 \\
\hline IV + V $\ldots$ & 2.449 & 1.149 & 46,92 & 8.242 & 2.650 & 32,13 \\
\hline Total & 69.598 & 3.892 & 5,59 & 96.632 & 6.130 & 6,34 \\
\hline
\end{tabular}

* Están incluidas las empresas que funcionaban el año 1925-26 completo.

Lista de indicadores:

1. Número de empresas comerciales.

2. Númeto de empresas comerciales que utilizan el trabajo asalariado.

3. Porcentaje de empresas comerciales que utilizan el trabajo asalariado.

4. Número de ocupados.

5. Número de asalariados, trabajadores y empleados.

6. Porcentaje de trabajadores y empleados.

FUENTE: El comercio y la industria privados en Ucrania, Járkov, 1929, tablas 96-124 (en ruso).

Es más cómodo presentar los datos sobre los cambios en las categorias de patentes en forma de una tabla que llamaremos 12 matriz de los pasos. Indica la dirección y la intensidad de desplazamientos entre las empresas comerciales durante 1925-26. En nuestro caso la matriz de los pasos está compuesta de seis líneas y seis columnas. Las cinco primeras caracterizan pasos de las empresas de una categoría a la otra, en la sexta línea está indicada la cantidad de empresas surgidas y en la sexta columna la de las desaparecidas. La suma en las líneas es igual al número de empresas de cada categoría para comienzos de 1925-26. La integran las empresas comerciales cerradas durante 1925-26. La suma en las columnas es igual a la cantidad de las empresas de cada 
categoria para comienzos de 1926-27. La integran las empresas que aparecieron durante 1925-26. Por ejemplo, a comienzos de 1925-26, en Ucrania funcionaban 34.027 empresas de primera categoría. Para 1926-27 su número alcanzó 40.685. En medio año 879 empresas de primera categoría pasaron a la segunda y 77 a la tercera. Aparecieron 20.252 empresas nuevas de primera categoria y desaparecieron al mismo tiempo 12.966.

La matriz de los pasos permite hacer varias observaciones. En medio año 4.350 empresas cambiaron de categoría (el 2,9 por 100 ). Son más móviles las empresas de IV y V categorias (cambiaron de categoría el 11,1 y 6,8 por 100 , respectivamente). Fueron mucho más estables las empresas de primera y segunda categorias (el 2,8 y 2,6 por 100, respectivamente, cambiaron de sus categorias). En total, predominaba la tendencia hacia el crecimiento de las empresas, aunque no se revelaba con toda claridad: el 2,1 por 100 de las empresas de segunda categoría elevaron su nivel y el 0,5 por 100 lo bajaron;
de las de la cuarta, el 6,6 y 4,5 por 100 . Las empresas comerciales de las dos primeras categorias poseian insignificantemente pequeña posibilidad de pasar a la cuarta o quinta categoría ( 0,1 por 100 ), igual que las de la cuarta o quinta prácticamente no pasaban a las dos primeras (probabilidad de tal paso era igual al 0,1 por 100). Las empresas comerciales de tercera categoria poseían idénticas posibilidades de pasar a cualquiera de los grupos opuestos.

\section{TABLA 2}

Matriz de los pasos de las empresas comerciales privadas durante 1925-26

\section{NÚMERO DE PASOS}

\begin{tabular}{|c|c|c|c|c|c|c|c|}
\hline \multirow{2}{*}{$1925-26$} & \multicolumn{7}{|c|}{ 1926-27 Categorias de patente } \\
\hline & $I$ & II & $I I I$ & IV & $V$ & Cerrodas & Total \\
\hline $\mathrm{I} \quad \ldots \ldots \ldots \ldots$ & 20.105 & 879 & 77 & 0 & 0 & 12.966 & 34.027 \\
\hline II $\ldots \ldots \ldots \ldots$ & 313 & 36.559 & 1.235 & 38 & 2 & 22.490 & 60.637 \\
\hline III $\ldots \ldots \ldots \ldots$ & 15 & 598 & 22.341 & 696 & 25 & 8.833 & 32.508 \\
\hline IV $\ldots \ldots \ldots \ldots$ & 0 & 4 & 171 & 1.980 & 258 & 1.517 & 3.930 \\
\hline $\mathrm{V}, \ldots \ldots \ldots$ & 0 & 0 & 7 & 32 & 240 & 294 & 573 \\
\hline Abiertas ...... & 20.252 & 30.593 & 10.893 & 2.221 & 389 & - & 64.348 \\
\hline Total $\ldots \ldots \ldots$ & 40.685 & 68.633 & 34.724 & 4.967 & 914 & 6.100 & 一 \\
\hline
\end{tabular}


II. PORCENTAjE DE PASOS

\begin{tabular}{|c|c|c|c|c|c|c|c|}
\hline \multirow{2}{*}{$1925-26$} & \multicolumn{7}{|c|}{ 1926-27 Categorias de patente } \\
\hline & $I$ & II & III & $I V$ & $V$ & Cerradas & Total \\
\hline$I \quad \ldots \ldots \ldots \ldots$ & 59,1 & 2,6 & 0,2 & 0 & 0 & 38,1 & 100,0 \\
\hline$n$ & 0,5 & 60,3 & 2,0 & 0,1 & 0 & 37,1 & 100,0 \\
\hline III $\ldots . . . \ldots \ldots$ & 0 & 1,8 & 68,7 & 2,1 & 0,1 & 27,2 & 100,0 \\
\hline IV $\ldots \ldots \ldots \ldots$ & 0 & 0,1 & 4,4 & 50,4 & 6,6 & 38,6 & 100,0 \\
\hline$v \ldots \ldots \ldots$ & 0 & 0 & 1,2 & 5,6 & 41,9 & 51,3 & 100,0 \\
\hline Abiertas ....... & 31,5 & 47,5 & 16,9 & 3,5 & 0,6 & - & 100,0 \\
\hline
\end{tabular}

FUENTES: El comercio y la industria privada en Ucrania, Járkov, 1929, tablas 96-124; Anuario estadistico 1925-26, Moscú, 1928, PP. 96-97, 104-105; Anwario estadistico 1926-27, Moscú, 1929, pp. 86, 92.

La matriz de los pasos demuestra lo inestable que eran las empresas privadas durante la NEP. Durante medio año desaparecieron el 35 por 100 de ellas. Al mismo tiempo, el proceso de surgimiento de nuevas empresas no sólo compensaba completamente las pérdidas, sino que aseguró el crecimiento de su cantidad en el 13,9 por 100. Las empresas de la tercera categoria fueron las menos amenazadas de desaparición, de las cuales el 68,7 por 100 conservaron su status, y tan sólo el 27,2 por 100 desaparecieron. Sobre las empresas comerciales de la quinta categoria pendía la mayor posibilidad de desaparecer. Más de la mitad de ellas dejaron de existir (véase tabla 2). Entre las empresas de las dos primeras categorías continuaban funcionando un 60 por 100 .

El análisis de la intensidad de pasos de las empresas urbanas y rurales demuestra que el comercio de ciudades pequeñas era el más estable (cambiaron de categoría el 2 por 100 de empresas), ocupando el polo opuesto, el más móvil, el comercio rural (cambiaron de cartegoria el 3,2 por 100 de empresas).

\section{EL PRONOSTICO RETROSPECTIVO DE LA ESTRUCTURA SOCIAL EN EL COMERCIO PRIVADO A COMIENZOS DE LOS AÑOS 30: MODELADO ALTERNATIVO}

¿Qué estructura social en el comercio privado podria configurarse hacia comienzos de los años 30 si hubieran continuado los procesos caraterísticos para la mitad de los 20? Para dar la respuesta a este interrogante proponemos 
una metodologia de pronóstico retrospectivo asentada en el modelado sobre la base de confrontación de los hechos (recordemos que para comienzos de los años 30 la actividad empresarial en la industria y el comercio fue prohibida).

Un instrumental adecuado para resolver el problema lo dan los modelos teóricos de probabilidades que utilizan las propiedades de los procesos de Markov (la clase de procesos casuales que recibió el nombre de ese matemático ruso, su inventor). Se utilizan ampliamente en las investigaciones de la dinámica social por ser los más simples entre los modelos que toman en cuenta las relaciones entre diferentes grupos sociales.

El modelo de movilidad social basado en las propiedades del proceso estacionario de Markov (cadena de Markov), se describe con el estado inicial del sistema (o sea, con las cantidades de grupos en el momento inicial) y con la matriz de probabilidades de pasos a cada y de cada grupo durante un "paso». El modelo de Markov permite, partiendo de la composición numérica de los grupos, y a base de una suposición sobre la estabilidad de la estructura de pasos, recibir la información acerca de los estados subsiguientes de la estructura ${ }^{4}$. El modelo que toma en consideración tanto los desplazamientos de las empresas comerciales de una categoría a la otra, como el proceso de surgimiento y desaparición de las empresas, se denomina modelo «abierto».

El modelo imitativo de Markov da posibilidad de determinar la estructura del sistema de comercio privado que podría configurarse, por ejemplo, hacia 1930 a condición de que se hubiera conservado la estructura de pasos tal como existía durante 1925-26. Para obtener el pronóstico retrospectivo el investigador debe indicar la dinámica de composición numérica de las empresas que surgen, puesto que la fuente presta información tan sólo de cómo se distribuyen por las categorías (a partir de los datos del año de «base»), pero su número total en cada paso del modelo debe determinarse por los razonamientos sustanciales. En esta situación es oportuno examinar las principales variantes posibles de desarrollo del proceso ("guiones»). Si, dadas las diferencias en significados numéricos de ciertos indicadores, todos ellos llevaran a unos resultados homogéneos, podrá sacarse la conclusión de que el proceso tiene una tendencia estable.

Téngase presente que hay cinco guiones basados en diferentes hipótesis sobre los factores que deben determinar el número de las empresas de comercio que se abren. La suposición más simple, durante cada año se abren una cantidad de empresas igual a la de aparecidas durante 1925-26 (A). Otra

4 D. J. Bartholomew (1970). 


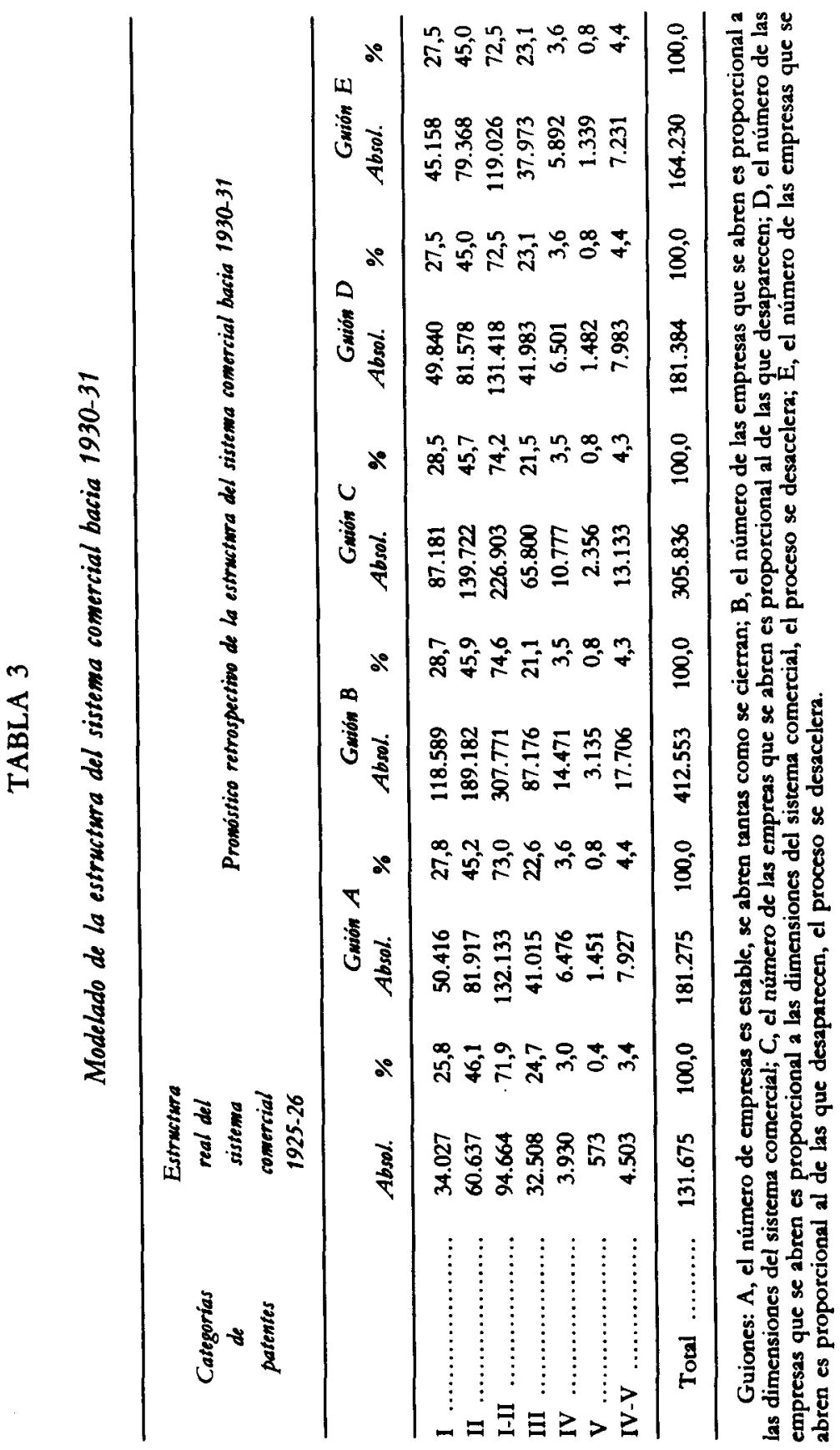


suposición, el número de empresas comerciales es proporcional a la envergadura del sistema de comercio (B). La tercera, el número de empresas comerciales es proporcional al de las que desaparecen, puesto que la cantidad de bancarrotas es un indicador de la coyuntura económica (C). El proceso de surgimiento de nuevas empresas en el segundo y tercero guiones puede llevarse a cabo a una velocidad decreciente, pues mientras el sistema de comercio se acerca a unas dimensiones óptimas desde el punto de vista económico, su crecimiento se hace cada vez más dificil (D, E ${ }^{5}$. De ahí que sea necesario examinar cinco variantes del modelado de cantidad de las empresas comerciales.

\section{TABLA 4}

Ritmo medio anual de crecimiento de las empresas comerciales privadas (en \%) (pronóstico retrospectivo a base del modelo de Markov)*

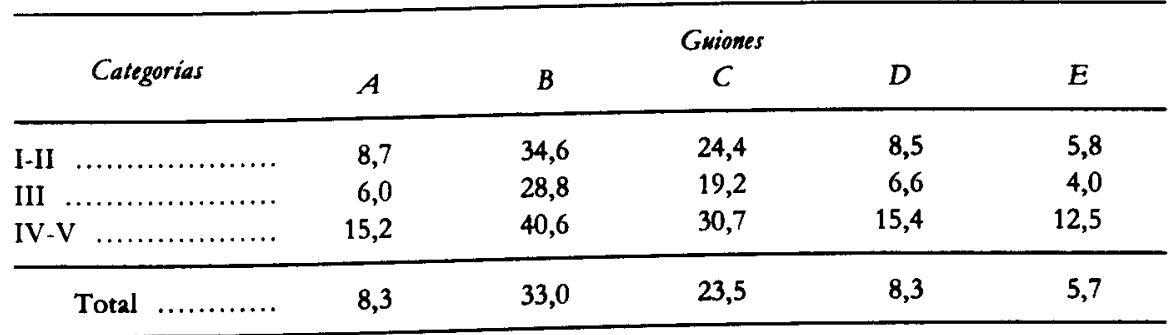

El siginificado de los guiones es igual al de la tabla 3.

* El ritmo medio de crecimiento se calcula según la fórmula: $\mathrm{T}=$ $Y_{n}$ es el significado del índice al año con el número ordinal $n$.

5 Las variantes indicadas de la dinámica de cantidad de las empresas comerciales que aparecen puede formalizarse del modo siguiente:
A) $\mathbf{X}_{t+1}=\mathrm{X}_{1}$
B) $\mathrm{X}_{\mathrm{t}+1}=\frac{\mathrm{X}_{1}}{\mathrm{~S}_{1}} \cdot \mathrm{s}_{\mathrm{s}}$
C) $X_{t+1}=\frac{X_{1}}{Y_{1}} \cdot Y_{1}$
D) $X_{t+1}=\frac{X_{1}}{S_{1}} S_{1 t}(1-L t)$
E) $X_{t+1}=\frac{X_{1}}{Y_{1}} \cdot Y_{1}(1-L t)$ 
Se ve claramente que diferentes guiones nos dan unos resultados que se difieren mucho. Con relación al modo de determinar la cantidad de las empresas nuevas en un modelo dado, pronosticada para 1930-31, el número de empresas comerciales varia de 164,2 mil a 412,6 mil (véase la tabla 3). Es sorprendente que en todos los guiones la estructura comercial resulta, en esencia, igual. La parte relativa de pequeñas empresas crece del 71,9 por 100 en 1925-26 hasta el $72,5-74,6$ por 100 en $1930-31$, mientras que la parte del grupo medio decrece del 24,7 hasta el $21,1-23,1$ por 100 y la parte relativa de las empresas de tipo capitalista aumenta del 3,4 hasta el 4,4 por 100 . A pesar de un torrente de empresas que se abren y desaparecen, la estructura comercial se queda estable. La trayectoria de los cambios se nota al analizar el ritmo medio anual de crecimiento. En los marcos de todos los guiones el crecimiento numérico de empresas pequeñas era 1,2-1,3 veces mayor, y de las grandes 1,3-1,5 veces mayor que de las medias (véase la tabla 4).

La especificidad del comercio urbano y rural se revela en los cambios numéricos de las empresas de diferentes categorias. Asi, de conformidad con el modelo, en el comercio rural crecerá el número de buhoneros (I categoría), al mismo tiempo aumentará rápidamente el pequeño comercio al por menor (IV categoría). En los centros urbanos locales el número de vendedores ambulantes y buhoneros disminuirá 1,4 veces, mientras que crecerá en la mayor medida el comercio de la $\mathrm{V}$ categoría (5,4 veces). Estas particularidades del desarrollo del comercio reflejan las diferencias en la organización de abastecimiento de la ciudad y el campo. La ciudad era centro del comercio al por mayor y de alli las mercancias llegaban al campo, donde, a su vez, pasadas por una red de pequeñas empresas mayoristas se repartian entre los mercaderes al por menor. La baja densidad de la población y el poder adquisitivo no muy alto de los campesinos llevaban a que en las aldeas podian trabajar con éxito bohuneros ambulantes o quienes cojugaban el comercio con la artesanía o la agricultura.

Los resultados del modelado demuestran que a mediados de los años 20 , en el sector privado se formaba una estructura social propia de la sociedad capitalista. Primero, para aquel entonces eran prácticamente imposibles ya los pasos del grupo inferior al superior y viceversa. Segundo, evanzaba el

donde $X_{t+1}$ es el número de las empresas comerciales que se abren durante $t+1$ años;

$Y$, es el número de empresas quebradas en $t$ años;

$S_{1}$ es a cantidad total de empresas comerciales en año t;

$X_{1} ; Y_{1} ; S_{1}$ son significados de corespondientes parámetros en el año inicial (de base);

$\mathrm{L}$ es el indicador del ritmo de disminución del crecimiento de las empresas comerciales nuevas (en el presente trabajo es igual al 10 por 100 anual). 
proceso de crecimiento de los grupos opuestos y disminuía la parte relativa del medio, aunque el número absoluto de propietarios de las empresas de III categoría continuaba creciendo.

Ya se mencionó más arriba que el modelo de Markov se basa en la suposición de que los pasos de las empresas de una categoría a la otra son estables. En otras palabras, la estructura del comercio privado hacia 1930-31 podría ser igual a la predecida por el modelo a condición de que se hubieran conservado las condiciones del segundo semestre de 1925-26, extraordinariamente propicias para el crecimiento del capitalismo brotado de la pequeña producción.

A finales de 1924-25 comenzó a aplicarse la llamada «nueva práctica comercial» para con el sector privado de la economía nacional. Esta práctica preveía levantar limitaciones infundadas para la actividad emprendedora, aumentar créditos para el comercio y la industria privados, ampliar los contactos del sector privado con la industria estatal. La unueva práctica comercial» fue una respuesta a los fenómenos críticos engendrados por la ofensiva contra el sector privado a finales de $1923-24$ y la primera mitad de 1924-25, que no fue preparada económicamente ${ }^{6}$. Durante 1924-25, el monto de los créditos bancarios creció de 21,3 millones a 52 millones de rublos. Además de los bancos, existian las Sociedades de Créditos Mutuos (SCM), que eran independientes y prestaban a los comerciantes privados y que estaban financiadas en más del 50 por 100 por el Estado. Mediante las SCM el sector privado obtuvo durante 1924-25 8,8 millones, y en 1925-26, 30,9 millones de rublos 7 . Se ampliaron los lazos económicos del comercio y la industria privados con el sector público. El peso de los comisionistas privados en las compras de lo producido por los trust se elevó hasta el 15 por 100 a finales de $1924-25^{8}$. En 1925-26, los comerciantes mayoristas reforzaron notablemente sus posiciones en el mercado de materias primas agrícolas y de artesanía. En resumen, la aplicación de la unueva práctica comercial» influyó positivamente en la economía nacional. Lo corrobora la disminución de los precios. Por ejemplo, el recargo comercial minorista del precio al por mayor disminuyó para los artículos industriales del 44,4 por 100 a comienzos de 1924-25 hasta el 26,3 por 100 al expirar aquel año 9 . El consumidor tenía que pagar ahora un 12 o 13 por 100 menos que hacía un año.

6 M. M. Zhirmunski (1927), pp. 129, 133-135; I. Mingulin (1927), p. 62; C. M. Kron (1926), p. 119.

7 I. Mingulin (1927), p. 63.
8 I. Mingulin (1927), p. 61.
' C. M. Kron (1926), p. 133. 
La gran afluencia del dinero y el abastecimiento ampliado llevaron a un rápido crecimiento del comercio privado. Su número creció para finales de 1925-26, comparado con el de la primera mitad de 1924-25, de $462,5 \mathrm{mil} \mathrm{a}$ 603,1 mil $(30,4$ por 100$)$, y la circulación, de $1.684,8$ millones hasta $3.368,1$ millones de rublos (100 por 100) ${ }^{10}$. Durante un año y medio el sector privado no cedió sus posiciones en el intercambio. Le pertenecia el 21-22 por 100 de operaciones en la circulación intermediaria del país, mientras que la parte de empresas privadas era igual al 79 por $100^{11}$. Hacia 1926-27, el comercio privado alcanzó el máximo nivel de su desarrollo. El número de las empresas era de 675,2 mil, siendo su circulación anual igual a 6.173,3 millones de rublos ${ }^{12}$.

El modelo imitativo presentado se construyó a base de la información sobre los desplazamientos de las empresas comerciales durante el período cuando era mayor la posibilidad de pasar al grupo de empresarios del tipo capitalista. De ahí que el modelo da el pronóstico de las dimensiones máximas posibles del grupo capitalista en el comercio. Si durante los cinco años subsiguientes se hubieran conservado las condiciones propicias para el desarrollo del sector privado, la parte relativa de las empresas de IV y V categorías habría alcanzado el 4,4 por 100 y el número de sus propietarios habria variado entre 7,2 mil y 17,6 mil personas, en función del guión examinado (tabla 3). Así que el ritmo de formación de los grupos opuestos en el comercio privado de los años 20 era bajo.

\section{EL MODELADO DE LA MOVILIDAD SOCIAL EN DIFERENTES SECTORES DEL COMERCIO PRIVADO}

Las indagaciones del sector privado comercial no deben perder del campo visual el hecho de que diferentes sectores del comercio estaban en condiciones diferentes. Para los empresarios que vendian comestibles, la fuente de abastecimiento era una miríada de pequeños productores. En esta área eran muy altas las posibilidades de utilizar la coyuntura del mercado, escapar del control y la reglamentación por parte de los órganos estatales. Los empresarios que se especializaban en la venta de artículos industriales eran abastecidos por los trusts y los sindicatos estatales, así como por las empresas industriales

10 Researcb in Economic History, Nueva York, 1991.

11 Ibidem.

12 Ibidem. 
privadas. Además, una parte considerable de las mercancias que pasaban por el comercio privado era elaborada por artesanos. Las posibilidades de controlar la actividad empresarial en el sector de venta de los artículos industriales era mayor, puesto que aqui, a diferencia del comercio de productos agrícolas, los comerciantes requerian suministros por parte de los trusts y los sindicatos. Los empresarios que se ocupaban de vender tejidos, dependian de la politica del Sindicato Textil Nacional (CTN) que era monopolista en su sector determinando los precios al por mayor, las condiciones de crédito, el surtido de tejidos.

A base de los materiales de la investigación se pueden separar los sectores que se ocupaban de vender comestibles, artículos industriales (salvo el comercio de tejidos) y el comercio de tejidos. En total, 61.260 empresas comerciales ( 88 por 100 del total de investigadas) formaron los tres grupos indicados.

En el presente artículo no se incluyeron los datos sobre el número de empresas de cada sector de comercio que se abrian y se cerraban; por esto el modelado se hacia partiendo de la suposición de que los pasos de los empresarios privados se limitaban con el cambio de categoría y el número total de empresas era constante. Este modelo (se llama «cerrado») es una aproximación grosso modo a los procesos reales. Sin embargo, permite revelar las tendencias en el cambio estructural del comercio. De acuerdo con el pronóstico retrospectivo, hacia el año 1930-31, el peso relativo de las empresas grandes en el comercio privado podría crecer del 2,9 hasta el 7,4 por 100 , y el de las pequeñas podría disminuir del 67,8 al 60,3 por 100 . La parte del grupo intermedio podría crecer insignificantemente (del 29,3 al 32,3 por 100). En total, se ve claramente una dependencia: cuanto mayor es la categoria de patente, tanto más rápido crece el número de empresas de este grupo (véase la tabla 5). De este modo, tanto el modelo que toma en cuenta el proceso de aparición y liquidación de las empresas, como aquel que se basa en la suposición de que su número sea invariable, nos presentan un cuadro cualitativamente único del desarollo del sector privado. Hace falta destacar que esto puede servir de prueba de fiabilidad de los pronósticos obtenidos.

El pronóstico retrospectivo construido a base del modelo de tipo «cerrado" demuestra que hacia 1930-31, en el grupo de sectores especializados en la venta de comestibles, el número de empresas comerciales de IV y V categorias podria crecer 4,4 veces, aumentándose su peso específico, en vista de ello, del 1,9 al 8,2 por 100. Al mismo tiempo, crecería del 24,6 al 27,8 la parte de empresas de III categoría y disminuiría la de las empresas de I y II categorías, del 73,6 al 64 por 100. En el grupo de sectores que se ocupaban de vender 
artículos industriales se llevarian a cabo procesos parecidos, aunque serían más flojos. La parte de empresas de IV y V categorías podria crecer del 2 al 4,5 por 100 , y de las de I y II categorias podría disminuir del 71,4 al 65,6 por 100 ), creciendo a la vez la parte de las empresas de I y II categorías (del 11,7 al al 25,5 por 100 ) (véase tabla 5 ).

sucederia con las empresas comerciales de III categoria (del 85,5 al 73,8 por 100), creciendo a la vez la parte de las empresas de I y II categorias (del 11,7 al 25,5 por 100 ) (véase tabla 5 ).

De esta suerte, el número de empresarios del tipo capitalista podria crecer notoriamente tan sólo en los sectores ligados con el espontáneo mercado campesino. En el comercio que se abastecía con los artículos de la industria estatal era prácticamente imposible el crecimiento del número de empresarios del tipo capitalista. Hasta en las condiciones de una disminuida presión fiscal, ampliada prestación de créditos y facilidades en el abastecimiento, el monopolio del sector estatal en la fabricación de varios tipos de artículos (por ejemplo, tejidos) permitía controlar el desarrollo de grupos correspondientes del sector privado. Por eso había posibilidades de realizar una política de regulación del comercio y la industria privadas no mediante proscripciones cada vez más severas, sino reforzando las posiciones económicas del sector socialista, elevando la eficacia de las empresas estatales.

\section{EL MODELADO DE LA ESTRUCTURA SOCIAL \\ EN EL SECTOR PRIVADO: RESULTADOS \\ DE UNA REGULACION ESTATAL «RIGIDA»}

Los cambios en la política del Estado soviético hacia la empresa privada se hicieron notorios ya en 1926-27. Se modificó la legislación fiscal aumentándose la parte de impuestos sobre las ganancias de los empresarios y comerciantes que utilizaban la mano de obra asalariada, del 29,6 al 46,6 por 100 . Esto llevó a que la parte de acumulación de la ganancia neta decayera del 28,9 al 13,1 por $100^{13}$. Esa plítica fiscal arruinaba a los empresarios privados. Miembros de una comisión especial para estudiar el sector privado de la economía nacional, encabezada por Sergó Ordzhonikidze, afirmaban que los oganismos financieros «llevaron la presión fiscal sobre el capital privado hacia uıa tensión extrema, hasta un límite tras el cual está la imposibilidad de realizar actividad económican ${ }^{14}$. El reforzamiento de la imposición fiscal y la

13 I. Mingulin (1927), p. 126.

14 I. Mingulin (1927), p. 127. 
aprobación de varios actos jurídicos que limitaban la actividad empresarial llevaron a que ya en 1927-28 el número de empresas privadas disminuyera en el 17,6 por 100 , y su circulación, en el 23,8 por $100^{15}$. El modelo construido a base de datos sobre los desplazamientos sociales de los comerciantes y empresarios durante $1926-27$ presta la posobilidad de analizar los cambios estructurales del sector privado en medio de una política fiscal rígida y de las limitaciones en el abastecimiento y la prestación de créditos. Para construir el modelo pueden utilizarse los datos sobre los impuestos sobre la renta que pagaban los empresarios privados cuya ganancia anual era mayor de 900 rublos ${ }^{16}$. Estaban sujetos a la imposición los gastos privados, los medios invertidos en bienes inmuebles o en la ampliación del capital fijo o circulante 17. El concepto "ganancia imponible», ampliamente utilizado por los órganos financieros, se acerca al concepto "ganancia neta» que se utiliza en las estadísticas.

\section{TABLA 5}

El modelado de la estructura de sectores del comercio privado bacia 1930-31 (modelo "cerrado»)

\begin{tabular}{|c|c|c|c|c|c|c|c|c|}
\hline \multirow[t]{3}{*}{ Categorias } & \multicolumn{4}{|c|}{ Todo el comercio } & \multicolumn{4}{|c|}{$\begin{array}{l}\text { Sectores del comercio que } \\
\text { se especializan en la venta } \\
\text { de productos agricolas }\end{array}$} \\
\hline & \multicolumn{2}{|c|}{$\begin{array}{c}\text { Datos } \\
\text { reales } \\
1925-26\end{array}$} & \multicolumn{2}{|c|}{$\begin{array}{l}\text { Pronóstico } \\
\text { retrospectivo } \\
1930-31\end{array}$} & \multicolumn{2}{|c|}{$\begin{array}{c}\text { Datos } \\
\text { reales } \\
1925-26\end{array}$} & \multicolumn{2}{|c|}{$\begin{array}{l}\text { Pronóstico } \\
\text { retrospectivo } \\
1930-31\end{array}$} \\
\hline & Absol. & $\%$ & Absol. & $\%$ & Absol. & $\%$ & Absol. & $\%$ \\
\hline I & 15.934 & 22,9 & 12.652 & 18,2 & 8.139 & 20,4 & 5.671 & 14,2 \\
\hline II & 31.235 & 44,9 & 29.298 & 42,1 & 21.167 & 53,1 & 19.840 & 49,8 \\
\hline I-II & 47.169 & 67,8 & 41.950 & 60,3 & 29.306 & 73,6 & 25.511 & 64,0 \\
\hline III $\ldots \ldots$. & 20.390 & 29,3 & 22.496 & 32,3 & 9.776 & 24,6 & 11.086 & 27,8 \\
\hline … & 1.827 & 2,6 & 3.438 & 4,9 & 663 & 1,7 & 1.809 & 4,5 \\
\hline $\mathrm{V}, \ldots \ldots \ldots \ldots \ldots \ldots \ldots$ & 212 & 0,3 & 1.740 & 2,5 & 76 & 0,2 & 1.415 & 3,7 \\
\hline IV $-V \quad \ldots \ldots \ldots \ldots \ldots$ & 2.039 & 2,9 & 5.178 & 7,4 & 739 & 1,9 & 3.224 & 8,2 \\
\hline Total & 69.598 & 100,0 & 69.598 & 100,0 & 39.821 & 100,0 & 39.821 & 100,0 \\
\hline
\end{tabular}

15 Researcb in Economic History, Nueva York, 1991.

16 Impuestos de oficio y sobre la renta, Moscú, 1927, p. 212.

17 Impuestos sobre la renta y de oficio, Moscú, 1927, p. 157. 


\begin{tabular}{|c|c|c|c|c|c|c|c|c|}
\hline \multirow[t]{3}{*}{ Categorias } & \multicolumn{4}{|c|}{$\begin{array}{l}\text { Sectores del comercio que } \\
\text { se especializan en la venta } \\
\text { de articulos industriales }\end{array}$} & \multicolumn{4}{|c|}{ Comercio de tejidos } \\
\hline & \multicolumn{2}{|c|}{$\begin{array}{c}\text { Datos } \\
\text { reales } \\
1925-26\end{array}$} & \multicolumn{2}{|c|}{$\begin{array}{l}\text { Pronóstico } \\
\text { retrospectivo } \\
1930-31\end{array}$} & \multicolumn{2}{|c|}{$\begin{array}{c}\text { Datos } \\
\text { reales } \\
1925-26\end{array}$} & \multicolumn{2}{|c|}{$\begin{array}{l}\text { Pronóstico } \\
\text { retrospectivo } \\
1930-31\end{array}$} \\
\hline & Absol. & $\%$ & Absol. & $\%$ & Absol. & $\%$ & Absol. & $\%$ \\
\hline $\mathbf{I} \ldots \ldots \ldots \ldots \ldots \ldots \ldots$ & 5.524 & 32,2 & 4.871 & 28,4 & 670 & 7,1 & 264 & 6,1 \\
\hline n.......... & 6.719 & 39,2 & 6.369 & 37,2 & 199 & 4,6 & 835 & 19,4 \\
\hline I-II $\ldots \ldots \ldots \ldots \ldots \ldots$ & 12.243 & 71,4 & 240 & 65,6 & 506 & 11,7 & 1.099 & 25,5 \\
\hline III $\ldots \ldots \ldots \ldots \ldots \ldots \ldots$ & 4.562 & 26,6 & 5.127 & 29,9 & 3.670 & 85,5 & 3.164 & 73,8 \\
\hline IV $\ldots \ldots \ldots \ldots \ldots \ldots \ldots$ & 290 & 1,7 & 410 & 2,4 & 82 & 1,9 & 32 & 0,7 \\
\hline $\mathrm{V}, \ldots \ldots \ldots \ldots \ldots \ldots$ & 50 & 0,3 & 368 & 2,1 & 38 & 0,9 & 0 & 0,0 \\
\hline IV-V $\ldots \ldots \ldots \ldots \ldots \ldots$ & 340 & 2,0 & 773 & 4,5 & 120 & 2,8 & 32 & 0,7 \\
\hline Total $\ldots \ldots \ldots \ldots$ & 17.144 & 100,0 & 17.144 & 100,0 & 4.295 & 100,0 & 4.295 & 100,0 \\
\hline
\end{tabular}

En 1927-28 fue investigado el sistema de impuestos sobre la renta, cuyos resultados vieron luz en varias recopilaciones de series editadas por el Comité Estatal de Planificación y la Dirección Central de Estadísticas de la URSS, "Estadisticas de Ucrania» ${ }^{18}$. Durante la investigación se recopiló la información sobre el número de contribuyentes registrados por los órganos financieros en 1927-28 que pagaban el mismo impuesto en 1926-27. La investigación abarcó, en diferentes categorias sociales y profesionales, del 96,3 al 83,6 por 100 de todos los contribuyentes. La tabla 6 demuestra las tendencias en los cambios de composición numérica de diferentes grupos de empresarios ocupados en el sector privado de la economia nacional. Sumando las columnas 2 y 3 (véase la columna 1) se obtiene el número de comerciantes y empresarios de categoria dada que eran contribuyentes del impuesto sobre la renta en 1926-27, y la suma de las columnas 3 y 4 (véase tabla 5) arroja el número correspondiente a 1927-28. Lamentablemente, es imposible confrontar los grupos de empresarios separados en las estadísticas de la imposición sobre la renta, con las categorías de empresas comerciales puesto que en primer caso la división está basada en los indicios sociales y profesionales, mientras que en el segundo, en las dimensiones de las empresas. Sólo en gran parte hipotéticamente se puede admitir que el grupo de mercaderes que trabajaban solos corresponde a los propietarios de empresas de I y II categorías, y de los comerciantes que trabajan con ayuda de miembros de sus

18 Estadisticas de Ucrania, Serie IV, Estadisticas financieras, t. 4, fascículo I, Järkov, 1928; fascículo 2, Járkov, 1928; fascículo 3, Járkov, 1930. 
familias a los propietarios de las empresas de III categoría mientras que los propietarios que utilizaban la mano de obra asalariada corresponden a los propietarios de las empresas de IV y V categorias.

Las estadisticas de los impuestos sobre la renta demuestran que el número total de comerciantes cuya ganancia superaba el mínimo no imponible creció durante 1926-27 el 8,3 por 100, el grupo de comerciantes que trabajaban con los miembros de sus familias creció el 10,2 por 100 y el grupo de mercaderes que trabajaban solos, el 9,1 por 100. Al mismo tiempo, el número de comerciantes que utilizaban mano de obra asalariada disminuyó el 6,1 por 100. Los datos de la tabla 6 indican una vez más que la composición numérica

\section{TABLA 6}

Comerciantes, empresarios y artesanos privados contribuyentes del impuesto sobre la renta en 1927-28

\begin{tabular}{|c|c|c|c|c|c|}
\hline \multirow[t]{2}{*}{ Categorias de empresarios } & \multicolumn{5}{|c|}{ Indicadores } \\
\hline & 1 & 2 & 3 & 4 & 5 \\
\hline \multicolumn{6}{|l|}{ COMERCIANTES } \\
\hline Que utilizan el trabajo asalariado ........ & 5.880 & 1.061 & 4.819 & 701 & 5.520 \\
\hline Que trabajan con su familia ............. & 14.185 & 788 & 13.397 & 2.243 & 15.640 \\
\hline Comerciantes individuales $. . . \ldots \ldots \ldots . . .$. & 70.554 & 14.550 & 56.004 & 20.974 & 76.978 \\
\hline Comerciantes en total $. . . . . . \ldots \ldots \ldots . . . .$. & 90.619 & 16.399 & 74.220 & 23.918 & 98.138 \\
\hline \multicolumn{6}{|l|}{ EMPRESARIOS } \\
\hline Que contratan a más de 3 trabajadores.. & 2.700 & 1.211 & 1.489 & 348 & 1.837 \\
\hline Que contratan a 3 y menos trabajadores. & 11.642 & $一^{*}$ & 11.642 & 5.457 & 17.099 \\
\hline $\begin{array}{l}\text { Total de empresarios que contratan la } \\
\text { mano de obra } \ldots \ldots \ldots \ldots \ldots \ldots \ldots \ldots \ldots\end{array}$ & & & 13131 & 5.805 & \\
\hline $\begin{array}{l}\text { mano de obra .............................. } \\
\text { Artesanos que trabajan con su familia. }\end{array}$ & $\begin{array}{r}14.342 \\
7.981\end{array}$ & $\begin{array}{l}1.211 \\
1.947\end{array}$ & $\begin{array}{r}13.131 \\
6.034\end{array}$ & $\begin{array}{l}5.805 \\
3.580\end{array}$ & $\begin{array}{r}18.936 \\
9.614\end{array}$ \\
\hline $\begin{array}{l}\text { Artesanos que trabajan con su familia } \\
\text { Artesanos miembros del artel .......... }\end{array}$ & $\begin{array}{l}7.981 \\
4.898\end{array}$ & 1.459 & 3.439 & 4.021 & 7.461 \\
\hline $\begin{array}{l}\text { Artesanos miembros } \\
\text { Artesanos solos .................... }\end{array}$ & 54.445 & 17.748 & 36.697 & 25.555 & 62.097 \\
\hline Total de empresarios y artesanos ....... & 81.666 & 22.365 & 59.301 & 38.806 & 98.108 \\
\hline
\end{tabular}

* La fuente no contiene datos sobre el número de empresarios que abandonaron la categoría de contribuyentes del impuesto sobre la renta.

Indicadores:

1. Número de empresarios contribuyentes del impuesto sobre la renta en 1926-27.

2. Número de empresarios que abandonaron la categoria de contribuyentes durante 1926-27.

3. Número de empresarios contribuyentes del impuesto sobre la renta en 1926-27 y 1927-28.

4. Número de empresarios imponibles por primera vez.

5. Número de empresarios contribuyentes del impuesto sobre la renta en 1927-28.

FueNTES: Estadisticas de Ucrania, Serie VI, estadisticas financieras, t. 4, fasc. 2, pp. 2, 10, 18; fasc. 3 , p. 2. 
de empresarios privados del periodo de NEP fue inestable en extremo. Sólo el 81,9 por 100 de comerciantes, que para 1926-27 obtenian una ganancia superior al mínimo no imponible, fueron registrados de nuevo entre los contribuyentes en 1927-28. Fue más estable el grupo de comerciantes que trabajaban con sus familias. El $\mathbf{9 4 , 6}$ por 100 se quedaron entre los contribuyentes. La composición del grupo de comerciantes individuales sufría los más rápidos cambios (el 79,3 por 100 se quedaron entre los contribuyentes).

Los datos a nuestra disposición permiten calcular la estructura del sector privado que podría configurarse hacia 1930-31 a condición de que hubieran continuado las tendencias en los cambios numéricos de los grupos de empresarios existentes en 1926-27. Para obtener el pronóstico retrospectivo se hace un recálculo del número de personas que abandonaron la categoria de contribuyentes, que se quedaron en ella y que pagan el impuesto por primera vez, a base de los datos del «año anterion, y para el primer paso del modelo del «año de base, 1926-27». El modelo demuestra que el número total de comerciantes que obtenian unas ganancias superiores al mínimo no imponible podría crecer el 41 por 100 durante cuatro años. También habria crecido el número de comerciantes que trabajaban con sus familias, mientras que el de los que empleaban el trabajo asalariado habría disminuido para 1930-31 el 20,1 por 100 . Esto podría llevar a una modificación estructural del comercio privado: disminución en cuatro veces del número de empresarios que utilizaban el trabajo asalariado, crecimiento (del 15,6 al 18,4 por 100) de los comerciantes que trabajaban con su familia y de los que trabajaban solos (del 77,9 al 80 por 100 ) (tabla 7).

El crecimiento numérico de empresarios se habría asegurado, en primer término, ampliándose el comercio privado urbano, que creció el 66,4 por 100 . El comercio rural prácticamente no creció. La especificidad del comercio rural y urbano se mostraba también en la dinámica numérica de diferentes categorias de empresarios. En el campo, hacia 1930-31 habria desaparecido, en esencia, el comercio contratando la mano de obra asalariada. En la ciudad creció rápidamente el grupo de comerciantes que trabajaban solos, mientras que en el campo este grupo disminuyó el 12,5 por 100 .

El análisis de los cambios numéricos del grupo de empresarios y artesanos pagadores del impuesto sobre la renta arroja resultados análogos. Aquí el pronóstico retrospectivo demuestra las tendencias siguientes. El número total de personas cuyas ganancias superaban el mínimo no imponible se duplicó durante un año. $\mathrm{El}$ número de artesanos individuales creció al ritmo más rápido (3,7 veces), siguiendo a aquéllos los empresarios que contrataban menos de tres personas ( 3,4 veces). Al mismo tiempo, el número de empresa- 


\section{TABLA 7}

El pronóstico retrospectivo del nümero de empresarios privados (a base de materiales obtenidos durante la investigación de los impuestos sobre la renta durante 1926-27)

\begin{tabular}{|c|c|c|c|c|}
\hline & \multicolumn{2}{|c|}{$1926-27$} & \multicolumn{2}{|c|}{$1930-31$} \\
\hline & Absol. & $\%$ & Absol. & $\%$ \\
\hline \multicolumn{5}{|l|}{ COMERCIO } \\
\hline $\begin{array}{l}\text { Comerciantes que contratan la mano de obra asala- } \\
\quad \text { riada } \ldots \ldots \ldots \ldots \ldots \ldots \ldots \ldots \ldots \ldots \ldots \ldots \ldots \ldots \ldots \ldots \ldots \ldots \ldots\end{array}$ & 5.880 & 6,5 & 4.700 & 1,6 \\
\hline Comerciantes que trabajan con su familia ........... & 14.185 & 15,6 & 23.448 & 18,4 \\
\hline 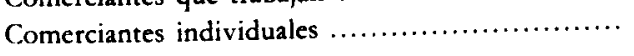 & 70.554 & 77,9 & 99.595 & 80,0 \\
\hline 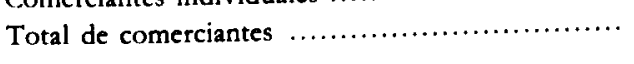 & 90.619 & 100,0 & 127.743 & 100,0 \\
\hline \multicolumn{5}{|l|}{ INDUSTRIA } \\
\hline Empresarios que contratan a más de 3 trabajadores. & 2.700 & 3,3 & 284 & 0,2 \\
\hline Empresarios que contratan a 3 y menos trabajadores. & 11.642 & 14,3 & 39.300 & 23,7 \\
\hline Artesanos que trabajan con su familia ............... & 7.981 & 9,8 & 15.675 & 9,5 \\
\hline 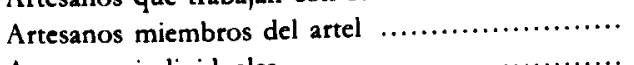 & 4.898 & 6,0 & 18.145 & 10,9 \\
\hline 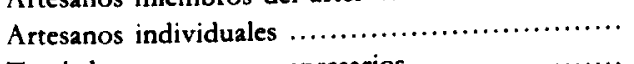 & 54.445 & 66,6 & 92.334 & 44,3 \\
\hline Total de artesanos y empresarios ... & 81.666 & 100,0 & 165.738 & 100,0 \\
\hline
\end{tabular}

rios que tenian más de tres trabajadores disminuyó 9,5 veces. En comparación con el comercio privado, en la producción creció más rápido el número de contribuyentes del impuesto sobre la renta y se revelaba con mayor exactitud el número decreciente de empresarios que contrataban la mano de obra.

De este modo, el modelo reconstruido a base de datos numéricos sobre los comerciantes y empresarios contribuyentes del impuesto sobre la renta en 1926-27 y 1927-28 demuestra que avanzaba el proceso de desintegración del grupo de empresarios del tipo capitalista. Se cerraban grandes empresas comerciales $e$ industriales basadas en la explotación de la mano de obra asalariada, y se robustecían las posiciones de los empresarios que sólo utilizaban el trabajo de miembros de sus familias. Así desaparecieron hasta los brotes más insignificantes de relaciones capitalistas configuradas hacia 1926-27, se formaba una estructura homogénea de producción privada, propia del momento inicial de la génesis capitalista. Dichos resultados reflejan la reacción del sector privado ante la dura politica económica del Estado soviético. 


\section{CONCLUSIONES}

El modelado matemático de desplazamientos sociales de los comerciantes y empresarios ha permitido dilucidar la tendencia motriz de los cambios estructurales del sector privado, y determinar los parámetros cuantitativos de los procesos indicados. Resultó que la diferenciación entre los productores pequeños durante la NEP tenía carácter inestable. Las posibilidades de acumular el capital eran en extremo limitadas por causa del desbarajuste total en la economía nacional, del bajo nivel de desarrollo de las fuerzas productivas, el muy bajo poder adquisitivo de la población. De ahi un ritmo lento de formación de la capa de grandes empresarios. Bastaba un empeoramiento insignificante de la situación económica para frenar ese proceso. Hasta en caso de crearse unas condiciones propicias para el avance del sector privado tendrian que pasar decenios para que los empresarios del tipo capitalista pudieran convertirse en una real fuerza económica.

Los resultados del modelado permiten sacar la conclusión de que la amenaza del crecimiento capitalista sobre la base de la pequeña producción fue en los años 20 sustancialmente menor de lo que suponían los dirigentes políticos y económicos del pais de entonces, y de lo que se sostiene tradicionalmente en las publicaciones científicas soviéticas. El proceso de diferenciación podría ser regulado con facilidad en los sectores donde el Estado ocupaba sólidas posiciones económicas. Por consiguiente, las medidas extraordinarias de finales de los años 20 que apuntaban a desplazar el comercio y la industria privados de la economía nacional fueron injustificadas económicamente.

\section{BIBLIOGRAFIA}

Bartholomew, D. J. (1970): Stochastic Models for Social Processes, Nueva York, Wiley. KRON, C. M. (1926): El comercio privado en la URSS (en ruso), Moscú, Birzha.

LENIN, V. I. (1975): Obras completas (en ruso), tomo 43, p. 61, 5." ed., Moscú, Politizdat.

Mingulin, I. (1927): Las vías de desarrollo del capital privado (en ruso), Moscú, NK RKI.

ZHIRMUNSKI, M. M. (1927): El capital comercial privado en la economía nacional (en ruso), Moscú, NFK. 